\title{
Face Photo Recognition using Sketch Image for Security System
}

\author{
A.Sivasangari, S.Poonguzhali, Immanuel Rajkumar, Maheshwari
}

\begin{abstract}
Because of expanding requests in application regions, for example, validation of law requirement, video reconnaissance, banking and access to security frameworks, programmed face acknowledgment has pulled in extraordinary consideration as of late. The acknowledgment of the face has ended up being one of the present essential applications. It is utilized fundamentally for computerized amusement, security and authorization. Face acknowledgment can be for the entire or fractional face, the entire face for the entire face identification and explicit highlights for the incomplete face discovery. The examination in most criminal cases relies upon the portrayals attracted by the observers ' depiction. The programmed recovery of photographs from the police mug-shot database that coordinate this depiction can conceivably diminish the quantity of suspects. Fundamentally, this procedure relies upon the onlooker and precision of the sketch craftsman's memory of the face to catch those subtleties. It can help scientists viably find or diminish potential suspects. By and large, be that as it may, a speculate's photograph picture isn't accessible and the best substitute is frequently a sketch drawing dependent on an observer's memory. We present another photograph recuperation framework utilizing face draws by changing a photograph picture into a sketch, fundamentally lessening the distinction among photograph and sketch, enabling the two to coordinate successfully.
\end{abstract}

Index Terms-Deep convolution Neural Network, Sketch Image and Data Augmentation.

\section{INTRODUCTION}

The acknowledgment of face photos is a standout amongst the most troublesome cenarios for heterogeneous face acknowledgment (HFR) including the examination of face pictures in various modalities [10, 11]. Calculations should likewise address mistakes in sketch pictures coming about because of inadequacies in memory and correspondence when an observer furnishes a sketch craftsman with a depiction of a suspect, notwithstanding the critical methodology hole $[12,13]$. These issues are causing conventional facial issues Recognition frameworks (FRSs) that perform ineffectively when the subject is distinguished in a sketch picture of a photograph exhibition, prompting the advancement of calculations explicitly intended to perceive the face photography [14]. Albeit a few strategies portrayed

Revised Manuscript Received on July 18, 2019.

A.Sivasangari, Dept of IT Sathyabama Institute of Sciece and Technology, Chennai,Tamilnadu, India (email: sivasangarikavya@gmail.com)

S.Poonguzhali, Dept of ETCE Sathyabama Institute of Sciece and Technology, Chennai, Tamilnadu, India (email: b.poonguzhali@gmail.com)

Immanuel Rajkumar, Dept of ECE Sathyabama Institute of Sciece and Technology, Chennai, Tamilnadu, India (email: imman047@gmail.com)

Maheshwari, Dept of CSE Sathyabama Institute of Sciece and Technology, Chennai, Tamilnadu, India in the writing purportedly accomplished high recuperation rates, these were normally accomplished by utilizing draws that are fundamentally the same as the first photographs (for example overlooking contortions brought about by memory and correspondence holes) and neglecting to recreate the mug-shot exhibitions kept up by law implementation offices utilizing a broad display.

\section{This letter's contributions are therefore:}

1) To maintain a strategic distance from the issue of a solitary sketch for every subject, a three - dimensional (3-D) morphable model is utilized to differ facial characteristics and incorporate another vast arrangement of pictures naturally.

2) The cutting edge profound system (pretrained on face pictures) for the acknowledgment of face photograph draws by means of exchange learning.

3) Since measurable portrays regularly contain a few errors, the engineered portrayals might be more amiable than the first sketch to the coordinating photograph. Indeed, execution is improved when different portrayals are utilized to look at the display pictures for each subject.

4) The combination of the proposed engineering with a main calculation demonstrates that the execution of both saw and measurable portrayals is additionally improved.

\section{RELATED WORK}

In writing, a few methods for face mind flight have been proposed, which incorporate intra-methodology meth-ods that change photographs and outlines so that examinations can be made inside a similar area. Brendan klair et al [1] proposed the frame work for local feature discriminant analysis and multi scale local binary patterns. Multiple projections are used for feature representation of minimum distance matching. Brendan Klair et al [2] proposed the heterogeneous face recognition involves matching two faces images from the alternate model such as infrared image or sketch image.

Shuxin at al [3] proposed method that used a database of 400 forensic sketches created in different time delays. In this database, they built a model to reverse the process. Galea et al [4] proposed the method that extracts multi scale local binary pattern descriptors from the overlapping patches for sketch image. Global and local texture information can be used for face photo sketch recognition. Unmistakable strategies incorporate Eigen-change, which completes union 
utilizing a direct mix of pictures, the expansion of Eigenpatches, which does combination at a nearby dimension, and the Bayesian system in, which considers the connection between neighboring patches for model structure. In an increasingly itemized audit of FH calculations can be found.

IState-of - the-craftsmanship flexibility techniques that le

arn or remove methodology invariant highlights incorporate the D-RS approach, which analyzes the descriptors of SIFT and MLBP separated from pictures containing three channels, the CBR method, which thinks about the highlights of MLBP extricated from individual facial segments, the FaceSketchID framework in, which consolidates D-RS with CBR, and the ongoing LGBP strategy in, which joins D-RS with CBR, and the ongoing LGBP strategy. The work done in is most applicable to this letter, yet the quantity of subjects and portrays utilized was restricted since the last were utilized physically made by utilizing a few craftsmen or programming administrators, making the procedure exorbitant and tedious. Christian et al[5] proposed a Malta software generated sketch database which contains the largest number of viewed software generated sketches. Wei Zhang et al [6] proposed automated face photo sketch recognition by developing advanced lassification algorithm to reduce the gap between the photos and sketches. Yaniv Taigman et al [7] proposed deep network which involves more parameters using locally connected layers without any sharing of weight. Sivasangari et al [9] proposed the crime reporting of integration of crime and complaint report for online crime registration for public.

\section{PROPOSED SYSTEM}

\section{A. Deep Convolutional Neural Network}

The proposed engineering comprises of a significant convolutionary neural system (DCNN), on which exchange learning is connected utilizing photographs and sketches 1 to empower the system to get comfortable with the the association between the two modalities. The decision to utilize a pre-prepared system as opposed to instating another one pursues perceptions in the writing that the previous approach will encourage quicker intermingling, relieve nearby minima and empower better speculation. The advantages of DCNN [9] are described as follows: 1) it was likewise intended to perceive faces, albeit just pictures, and 2) it is one of the main FRSs for unlimited face acknowledgment. It in this manner gives a decent beginning stage from which the parameters for face photograph sketch perceiving can be balanced. For system preparing and testing, a comparative usage procedure is connected to[ 9], specifically bootstrapping the DCNN for characterization pursued by triple installing to empower check and scaling the test pictures to three sizes to permit multi-scale testing.

\section{B. Data Augmentation}

Deep models, for example, the VGG-Face arrange, have ended up being more dominant than shallow systems and are subsequently significant for good performance. Such systems, nonetheless, contain a substantial number of trainable parameters and will in general be overfitted. Such systems should along these lines be prepared in an expansive number of classes, yet in addition in countless for each category[ 8]. Conventional errand databases, Article and face acknowledgment are frequently built utilizing the various pictures accessible on the web, however portrays and comparing photographs are infrequently accessible and just a single sketch for every subject is generally accessible, all things considered, databases and in openly accessible databases. Subsequently, notwithstanding the first photographs and draws, the 3-D Morphable model2 in permits the variety of the qualities for each face picture age and incorporates another arrangement of pictures to prepare the system.Both individual facial attributes (eyes, nose, mouth and face) and worldwide qualities (weight, age, tallness, stature, and so forth. The subsequent strategy is known as the DEEP (face) photograph - sketch framework (DEEPS) for an aggregate of 645 pictures (counting the first).

\section{Multiple Synthetic Sketches for Recognition}

Albeit manufactured pictures help to learn by enabling the DCNN to be adaptable for contrasts in the facial characteristics of pictures and draws, they can likewise be valuable in deciding the personality of a subject amid testing. This exploits the perception that trait modifications can deliver a sketch that balances the twists and distortions ordinarily found in representations contrasted with the comparing photographs. A case of this present reality is appeared in Fig. 1. For each subject, the attributes of a subset of 199 manufactured representations and the first are determined. Since a sketch is contrasted with the photographs of the exhibition, 200 dis-tance measures are shown in the display for each subject. These separations are joined with: 1) the middle of the main 49 matches and the match with the first sketch, and 2) the best match between nine portrayals and the first. Two separation esteems are therefore acquired for every examination of a sketch with a photograph, that are consolidated utilizing min-max standardization and entirety of-scores combination.

Because of their substantial amount, saw portrays are utilized to approve the execution of DEEPS in regard of a few writing proposed al-gorithms. Specifically, the mainstream CUFS database, which contains 606 subjects in the AR, XM2VTS and CUHK students, together with the portrayals of 946 subjects in the CUFSF database and the comparing pictures in the FERET shading database. While most works utilize just CUFS, its representations look somewhat like the comparing photographs and hence show the memory and correspondence holes insufficiently. In any case, the CUFSF outlines were made to purposefully contain a few twists and shape distortions to emulate true portrays Empirically, it was additionally seen that there are a few situations where LGMS performs discernibly more awful than DEEPS, and bad habit versa.This wonder likewise remains constant for the criminological portrayals and to decrease blunder rates by $756.7 \%$ and $80.7 \%$ contrasted with DEEPS and LGMS, separately. 


\section{Proposed Method}

The input is taken as image, if video is taken then later it is parsed into different frames of that video-images.Hence a Login HTML page for video upload and parsing the video into Image has been created. For output the Microsoft SQL database engine is taken for login details. Therefore, a login HTML page, a video upload page and parsing the video image page has been created. The Microsoft SQL database engine is used for login details. Once the table has been created, insert values into it. After the connection is established. While the program is running, it returns. Which should then be opened in a browser to login after uploading a video and finally scanning it to get the images scanned.

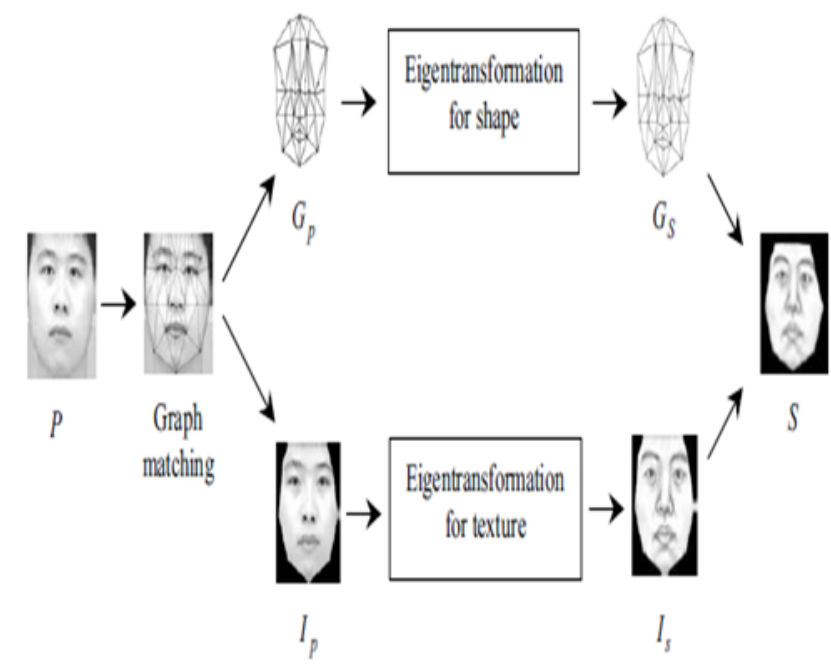

Fig1 Framework of the Face Sketch Synthesis System

\section{Bayesian Classifier}

Bayesian classifier can be used for reduce the effect of transformation error at the recognition stage. Bayesian recognition defines the difference between two faces as variation of intrapersonal or extrapersonal ie caused by individual difference. The proposed classification is based on intrapersonal method. Principal component analysis is applied to this set which consists of change mistake between blended sketch and genuine sketch. In our work, we separate the face image into shape and texture. It consists of two phases such as preparation and run time

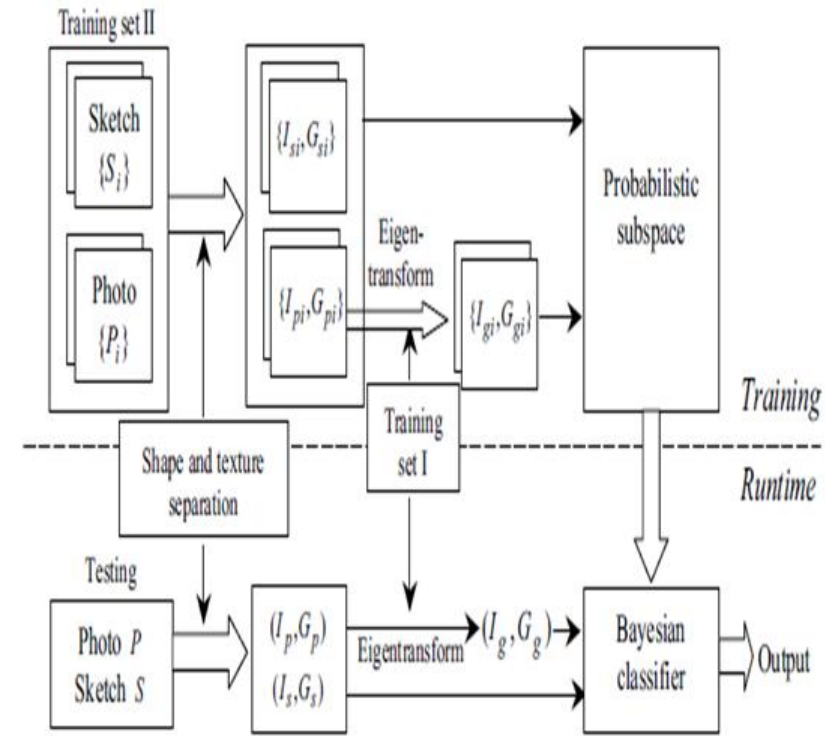

Fig.2 Framework of the Face Sketch Synthesis System

The faces image is converted in to sketch and coverted in to eigen face by considering the intensity of the image. The sketch is compared with sketch which is generated from criminal record of police. The face region is divided into overlapping patches. If two photo patches are similar,their sketch patches also similar. We separate the face image into shape and texture. In training set, photo to sketch eigen transform co efficient are computed. Photos and sketches are separated into shape and texture. Photo texture and shape in training set are transformed to pseudo sketches. The texture and shape sub spaces are calculated from training sketches and pseudo sketches.

\section{RESULT}

The PRIP-HDC dataset is utilized to assess the strategies utilized in certifiable pictures, which contain hand-drawn forensic portrays of 47 subjects made in true examinations from onlooker accounts. After the suspects in the sketches were identified, the mug-shot pictures were available [ 1], [ 2]. All subjects were only tested using the same models trained on the video, due to the dataset's small size. Additionally, thus customary execution measures may yield off base outcomes and therefore examination is performed utilizing the positions at which calculations recover each subject specifically. Extra outcomes are likewise benefit capable as a major aspect of the Supplementary Material.

\section{CONCLUSION}

This letter introduced a face photograph sketch acknowledgment framework using a 3-d morphable model to change facial highlights and naturally create new pictures, bypassing the issue of having just a solitary sketch for each subject in this manner empowering a profound convolutional neural system to get familiar with the connections among photographs and draws and surpass the execution of driving techniques. The blend of various representations at test-time was likewise appeared to result 
in improved execution for true outlines, since the facial component varieties can yield draws that are more like the coordinating photograph than the first sketch. Consequently, this is one of just couple of works considering profound learning for hand-drawn face outlines and various representations for subject distinguishing proof.

\section{ACKNOWLEDGMENT}

We wish to acknowledge the Department of Science and Technology, India and School of Computing, Sathyabama Institute of Science and Technology, Chennai for providing the facilities to do the research under the DST-FIST Grant Project No.SR/FST/ETI-364/2014.

\section{REFERENCES}

1. B. Klare, Z. Li, and A. K. Jain, "Matching forensic sketches to mug shot photos," IEEE Trans. Pattern Anal. Mach. Intell., vol. 33, no. 3, pp. 639- 646, Mar. 2011.

2. B. Klare and A. K. Jain, "Heterogeneous face recognition using kernel prototype similarities," IEEE Trans. Pattern Anal. Mach. Intell., vol. 35, no. 6, pp. 1410-1422, Jun. 2013.

3. S. Ouyang, T. M. Hospedales, Y. Z. Song, and X. Li, "ForgetMeNot: memory-aware forensic facial sketch matching," in Proc. Int. Conf. Com-put. Vis. Pattern Recognit., Jun. 2016, pp. 5571-5579.

4. C. Galea and R. A. Farrugia, "Face photo-sketch recognition using lo-cal and global texture descriptors," in Proc. Eur. Signal Process. Conf., Budapest, Hungary, Aug. 2016, pp. 2240-2244.

5. C. Galea and R. A. Farrugia, "A large-scale softwaregenerated face com-posite sketch database," in Proc. Int. Conf. Biometrics Special Interest Group, Sep. 2016, pp. $1-5$.

6. W. Zhang, X. Wang, and X. Tang, "Coupled information-theoretic encod-ing for face photo-sketch recognition," in Proc. IEEE Conf. Comput. Vis. Pattern Recognit., 2011, pp. 513-520.

7. Y. Taigman, M. Yang, M. Ranzato, and L. Wolf, "DeepFace: closing the gap to human-level performance in face verification," in Proc. IEEE Conf. Comput. Vis. Pattern Recognit., Jun. 2014, pp. 1701-1708.

8. F. Schroff, D. Kalenichenko, and J. Philbin, "FaceNet: A unified embed-ding for face recognition and clustering," in Proc. IEEE Conf. Comput. Vis. Pattern Recognit., Jun. 2015, pp. 815-823.

9. Infanta Amirtha Mary, N., Dharshini, J., Sivasangari, A.," Crime reporting integration of crime \& complaint reporting and effective data sharing with multi user access “,Vol.8,pp. 11916-11924.

10. Dr.R.Subhashini, E.Nagarajan and Niveditha.P.R, "Detection of an Incognitos Intruder in Industries and Semantic Mapping Of Emotions", International Journal Of Applied Engineering Research, ISSN-0973-4562, VOLUME 9, Number 20, 2014.

11. Sethuraman, R., Vaitheeswaran, E. "Student monitoring using opencv" International Journal of Applied Engineering Research Volume 9, Number 20 (2014) pp. 7411-7418

12. Saravanan, M, Sukanya, S, Image based password authentication system for banks, International Conference on Information Communication and Embedded Systems, ICICES 2017.

13. Harikrishnan Natarajan, Ajitha P,"An adaptive approach for Dynamic Resource Allocation in Cloud service", International Journal of Control theory and applications, vol 9(10), PP.4871-4878, 2016.
14. Sai, M.Y., Prasad, R.V.C., Niveditha, P.R., (...), Vigneshwari, S., Gowri, S., Low cost automated facial recognition system , Proceedings of the 2017 2nd IEEE International Conference on Electrical, Computer and Communication Technologies, ICECCT 2017. 